\title{
Function and structure in glycine receptors and some of their relatives
}

\author{
David Colquhoun and Lucia G. Sivilotti \\ Department of Pharmacology, University College London, Gower Street, London WC1E 6BT, UK
}

\begin{abstract}
In the field of ligand-gated ion channels, recent developments, both in the knowledge of structure and in the measurement of function at the single-channel level, have allowed a sensible start to be made on understanding the relationship between structure and function in these proteins. In this review, the cases of glycine, nicotinic ACh and glutamate receptors are compared and contrasted, and problems such as how binding of agonist causes the channel to open, and why partial agonists are partial, are considered. Some observations, both structural and functional, suggest that more attention needs to be paid to conformational changes that occur before the channel opens. Such changes might account for the interaction found between subunits of the glycine receptor while it is still shut and, perhaps, the agonist-dependent structural changes seen in AMPA receptors. They might also complicate our understanding of the binding-gating problem.
\end{abstract}

'Francis Crick...said that in the pioneering days of structure determination researchers were driven by the conviction that once they had solved a biological structure, its function or mechanism would become immediately obvious. It came as a shock when they found this was not necessarily so and that the opposite was more frequently true.' [1]

This review is a biased discussion of some recent work on glycine receptors, with reference to some other receptors where it seems appropriate, and where length allows. The aim is to discuss what can be learned about reaction mechanisms from single-channel analysis, and the extent to which it can be related to structure [2-4]. Desensitization is not considered owing to lack of space. As will be seen, it appears that, despite big advances in both areas, the link between them is still weak.

\section{What is known about structure? \\ Nicotinic ACh receptors}

Nicotinic ACh receptors have five subunits (two $\alpha$ and three non- $\alpha$ ) arranged quasi-symmetrically around the channel. Our knowledge of structure comes mainly from the electron microscopy work by Unwin and co-workers on Torpedo receptors $[5,6]$, and from the crystal structure of the Lymnea stagnalis ACh-binding protein [7,8]. The latter is a soluble pentamer of five identical subunits, each with 210 amino acids (less than half the 437 residues

\footnotetext{
Corresponding author: David Colquhoun (d.colquhoun@ucl.ac.uk).
}

of the human $\alpha 1$ subunit), and with $20-24 \%$ sequence identity with the extracellular domain of nicotinic subunits. Unwin's work (resolution up to $4 \AA$ ) is still the only source of structural knowledge about the four receptor transmembrane domains (M1 to M4) which form the channel, or about heteromeric receptors (Figure 1).

\section{Glycine receptors}

Glycine receptors are pentamers formed either from $\alpha$ subunits alone, or from both $\alpha$ and $\beta$ subunits. Heteromeric glycine receptors have subunit composition $\alpha_{3} \beta_{2}$ [9-11] and three agonist-binding sites, rather than the two sites found in nicotinic $\mathrm{ACh}$ or $\mathrm{GABA}_{\mathrm{A}}$ receptors. The homomeric glycine receptor is discussed later. There is no direct evidence about the structure of glycine receptors, so structural inferences are based solely on analogy with nicotinic receptors.

\section{Glutamate receptor family}

The long-running controversy about whether glutamate receptors have four or five subunits is now settled beyond reasonable doubt in favour of four. In the NMDA-type receptor there are two NR1 subunits each of which binds one glycine molecule, and two NR2 subunits that bind glutamate $[12,13]$. They are probably arranged as a dimer of dimers (one NR1 dimer and one NR2 dimer [14]).

The agonist-binding region is thought to be made up of a 'clamshell' structure that is formed from two disjoint extracellular parts of the receptor - the $\mathrm{S} 1$ region which is $\mathrm{N}$-terminal of M1, and the S2 region that is C-terminal of M2 (Figure 2). Crystal structures have been determined for a protein construct made by joining the S1 and S2 regions with an artificial linker. This has been achieved both for the GluR2 (AMPA-type) receptor [15] and for the NR1 (glycine-binding) subunit of the NMDA receptor [16]. As for the nicotinic receptor, atomic level resolution is available only for (part of) the extracellular domain, without its normal connection to the channel.

\section{Relating structure and function}

The link between agonist binding and opening of the channel

The current structural knowledge of the transduction mechanism is restricted largely to the nicotinic receptor, and even in that case, it is still relatively speculative. Unwin's view is summarized in Box 1.

Crystallographic data give us only a static picture of the receptor. An ambitious functional approach to the problem 


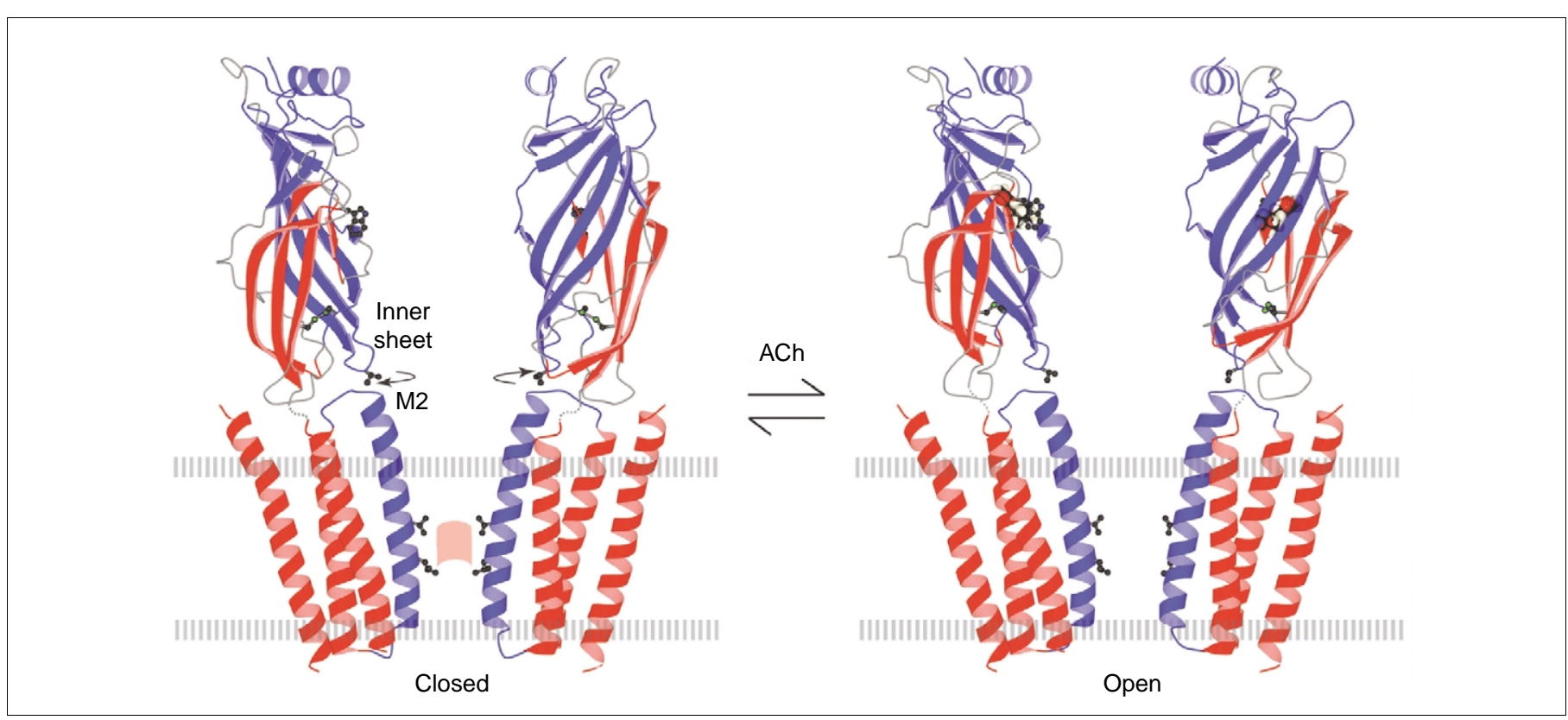

Figure 1. Transduction in the nicotinic ACh receptor. The closed-to-open transition for the Torpedo nicotinic channel is shown as envisaged by Unwin and co-workers [5]; the two $\alpha$ subunits in the receptor pentamer are in the cell membrane (grey). ACh binding produces a conformation change of the outer $\beta$ sheets of the extracellular domain (red) and a clockwise rotation of the inner $\beta$ sheets (blue, arrow). This motion of the extracellular domain is transmitted to the pore-lining M2 helix (blue), which moves to break the side-to-side hydrophobic interactions in the channel gate (pink) and open the channel. Reproduced, with permission, from Ref. [5].

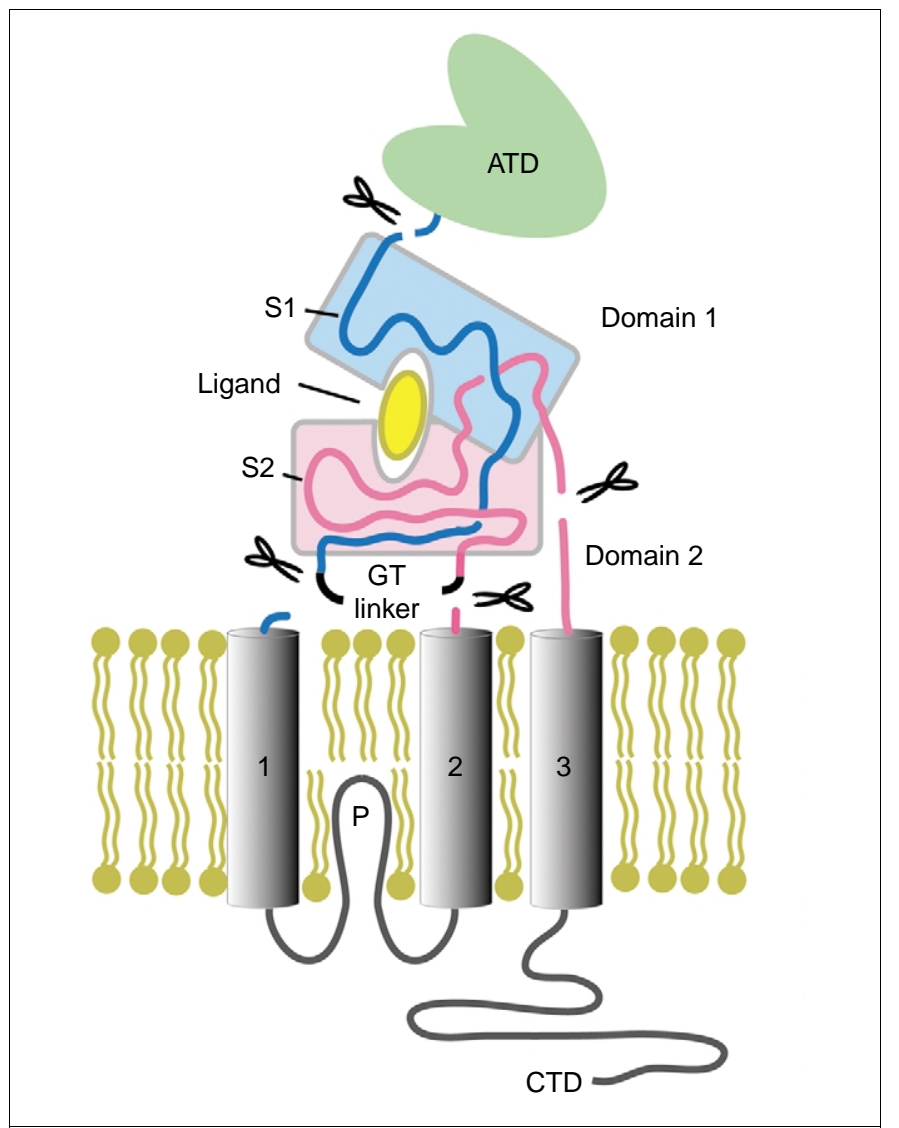

Figure 2. Layout of the AMPA receptor binding site. The binding site is formed from the discontinuous S1 and S2 domains, which form a 'clamshell' structure. The scissors symbols and the Gly-Thr (GT) linker show the construction of the soluble form of the binding-site domain used in the crystallographic studies by Gouaux and collaborators. The membrane domains are labelled $1, P, 2$ and 3 (sometimes called M1, M2, M3, M4). Abbreviations: CTD, C-terminal domain; ATD, $\mathrm{N}$-terminal domain. Reproduced, with permission, from Ref. [59] (c) (2004) Blackwell Publishing. of how the different protein domains move upon activation is analysis of 'linear free energy relationships' in nicotinic receptors [1,17-19]. These studies found that mutations that affect gating (i.e. that change the equilibrium constant for the open-shut conformation change, $E=\beta / \alpha$ ) do so mainly by changing the rate constant for channel opening $(\beta)$ when the mutation is near the binding site, but mainly by altering the closing rate constant $(\alpha)$ when the mutation is deep in the pore. This suggests that the transition state for the conformation change is 'open-like' in the binding region but 'shut-like' further down in the pore. Insofar as this can be interpreted in terms of a temporal sequence, this means that the perturbation following agonist binding starts near the binding site and propagates down towards the M2 channel-lining region. Although this seems almost self-evident, the method has the potential to track subtler aspects of the conformation change; for example Cymes et al. [18] conclude that the whole M2 helix (in the $\delta$ subunit) does not move synchronously but that the outer parts move before the inner parts.

\section{Measurement of function: binding and gating}

To understand how binding of agonist leads to opening of the channel, the first step must be to measure separately the initial binding of the agonist to the shut receptor, and the effectiveness of bound agonist in opening the channel. Making this distinction is the so-called 'binding-gating problem' [20]. In most cases, it can be solved only by singlechannel methods because whole-cell measurements of agonist potency $\left(\mathrm{EC}_{50}\right)$ cannot give us the separate physical constants for binding or gating.

Newer methods for such single-channel analysis make optimum use of all information in the record by fitting a mechanism to the whole sequence of openings and closings (rather than fitting time constants empirically to different 


\section{Box 1. Transduction: from binding to gating in the nicotinic $\mathrm{ACh}$ receptor channel}

Clues to what happens when ACh binds nicotinic receptor channels have come from comparison of Torpedo receptors with the ACh-binding protein $[7,8,58]$. In the latter, all five subunits (when a molecule of HEPES buffer, rather than ACh, is bound) have the same conformation, which is similar to that of the non- $\alpha$ subunits of the Torpedo receptor. In the unoccupied receptor, $\alpha$ subunits have a different conformation from 'non- $\alpha$ ' subunits, but convert to this 'non- $\alpha$ ' conformation once ACh is bound. This change (Figure 1 of main text) involves a displacement in the C-loop (the domain that lies over the binding pocket and contains the two adjacent cysteine residues) and a $15^{\circ}$ clockwise rotation of the inner extracellular $\beta$ sheets. As a result, the whole receptor becomes more symmetrical. Recent data on the structure of the ACh binding protein with nicotine or carbamylcholine bound confirm that the C-loop closes on the bound agonist [8], but do not show any difference in the position of the inner $\beta$ sheets between the structure with HEPES and those with agonists. This might be due to the absence of the channel domain or to the fact that we do not have an unliganded structure for comparison (even the HEPESbound structure could be an 'activated', or desensitized, state).

Although the details are far from certain, it has been suggested [6] that the bottom of the inner $\beta$ sheets of the extracellular domain contacts the channel-forming domains at the short extracellular loop that links M2 and $M 3$, in a way that is not the same for all subunits [60]. This linker could transmit the clockwise rotation of the extracellular inner part of the $\alpha$ subunits to their pore-lining M2 helices. This rotation could then destabilize the gate of the channel, a hydrophobic girdle at the narrowest point of the closed pore (the residues at positions $9^{\prime}, 10^{\prime}$,

dwell-time distributions, as was done originally [21,22]). Such methods have been developed by Qin et al. [23] (QUB program) and by Colquhoun et al. [24] (HJCFIT program). HJCFIT has been tested by simulation [25]; the predictions of the correlations between open and shut times (which are seen in all the channels discussed in this review) are compared with observations, to provide another criterion for distinguishing mechanisms [26]. Both programs have been used to analyze the mechanisms of the muscle nicotinic receptor [3,27,28]. HJCFIT has also been used to investigate mechanisms of glycine receptor activation [29,30]

Such analysis requires that a reaction mechanism be specified, and if the results are to be of much interest, this mechanism must describe physical reality (to a sufficient approximation). It must describe actual structural events if function is to be related to structure. Plausible attempts have been made for the muscle nicotinic receptor and some glycine receptors, but $\mathrm{GABA}_{\mathrm{A}}$, and especially glutamate, receptors have proved to be more difficult.

All of these receptor types have more than one binding site, and for both nicotinic and glycine receptors, the more ligand molecules that are bound, the more the channel tends to open (the efficacy $E=\beta / \alpha$ is larger, where $\beta$ is the opening rate constant and $\alpha$ is the shutting rate constant; Figure $3 \mathrm{a}$ ). Beyond that, there are substantial functional differences between nicotinic and glycine receptors. The prototype mechanism for a nicotinic receptor invokes two sequential binding events (Figure 3a), with opening possible from either 'monoliganded' or 'diliganded' receptors [21]. Opening can also occur without ligand binding although, except for some mutant receptors, such events are too rare to be analyzed. The diliganded open-state nicotinic receptor $13^{\prime}$ and $14^{\prime}$ of M2). In nicotinic receptors, the loss to the girdle of the M2 helices of the two $\alpha$ subunit destabilizes the gate sufficiently to produce concerted collapse of the remaining (non- $\alpha$ ) M2 domains (animation at http://www.ucl.ac.uk/pharmacology/dc.html\#movie). In the glycine receptor, disruption of three, rather than two, of the pore-lining M2 domains seems to be needed [29].

This picture is far from definite but its main features are supported by other lines of evidence. Various mutations in the M2-M3 linker of the $\alpha$ subunits of nicotinic or glycine channels impair channel function, probably by acting on gating [61-64], but have no effect if inserted in the non- $\alpha$ subunits of muscle nicotinic and glycine receptors $[61,65]$.

A gate-forming role for the hydrophobic residues in the middle of M2 is confirmed by the enhanced agonist sensitivity of macroscopic currents produced by $9^{\prime}$ hydrophilic mutations in all receptors in the superfamily [66]. This enhancement is seen irrespective of which ( $\alpha$ or non- $\alpha$ ) subunit carries the mutation $[11,65,67-69]$. Of course, these are results from recording whole-cell currents and cannot tell us whether the change in the $\mathrm{EC}_{50}$ measurement of agonist potency results from changes in binding or in gating. Single-channel work shows that mutations throughout M2 can affect gating: Cymes et al. [18] found that the greatest changes in the gating equilibrium constant $E$ are seen for mutations at positions $12^{\prime}, 13^{\prime}$ and $17^{\prime}$ in M2. However, evidence against a gate in the middle of $\mathrm{M} 2$ comes from substituted-cysteine accessibility experiments, which suggest that the narrowest point of the closed channel, and hence the gate, is towards the intracellular end of M2, at positions $-4^{\prime}$ to $2^{\prime}$ [70].

has high affinity for ACh: dissociation of $\mathrm{ACh}$ from it occurs at a slow, but measurable, rate [31].

\section{Are the binding sites the same in the resting state?}

In the (adult) nicotinic receptor, the subunits are arranged (anticlockwise) as $\alpha \varepsilon \alpha \delta \beta$, with binding sites at the $\alpha \delta$ and $\alpha \varepsilon$ interfaces (these are called the $a$ and $b$ sites in Figure 3). Good fits are obtained with mechanisms that assume that these two sites are different in the resting state. The extent of the difference in the affinity for ACh varies depending on species [27,28,32-36]. A standard mechanism with two different sites (representing $\alpha \delta$ and $\alpha \varepsilon$ sites) is shown in Figure $3(b-d)[22,37]$. Good fits can be obtained using the assumption that the two different sites do not interact (i.e. that binding to the $a$ site does not depend on whether the $b$ site is occupied, and vice versa).

In contrast to nicotinic receptors, observations on glycine homomeric $\alpha 1$ receptors $[29,30]$ cannot be fitted well with initially different independent binding sites. They can, however, be fitted if the binding sites are supposed to be initially identical but are able to interact while the channel is still shut (as will be discussed later). In neither case, therefore, can results be fitted with the standard Monod-Wyman-Changeux scheme [38], which assumes identical, non-interacting sites.

\section{Do the binding sites interact while the channel is still shut? Is there a conformation change before the channel opens?}

In the scheme shown in Figure 3(a), the possibility arises that the affinity (in the shut state) for the second binding event (equilibrium constant $K_{2}=k_{-2} / k_{+2}$ ) might not be the same as that for the first binding event (equilibrium constant $K_{1}=k_{-1} / k_{+1}$ ), even though the sites are initially 
(a)

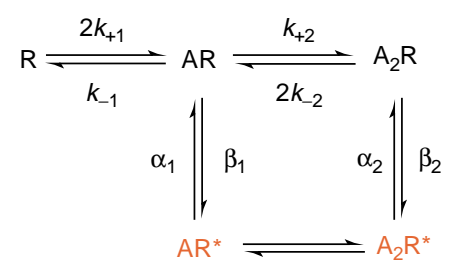

(c)

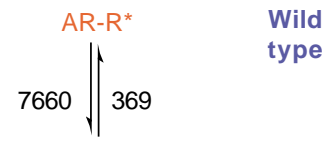

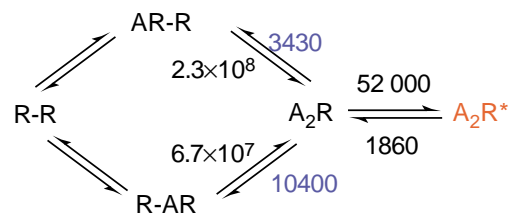

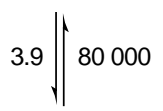

$\mathrm{R}-\mathrm{AR}^{*}$

$K_{\mathrm{a}}=155 \mu \mathrm{M} ; K_{\mathrm{b}}=14.7 \mu \mathrm{M} ; E=27.9$ (b)

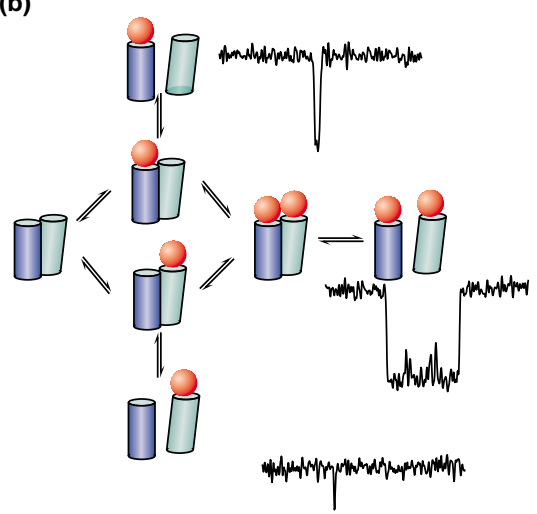

(d)

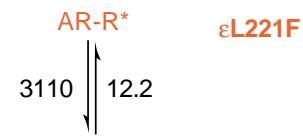

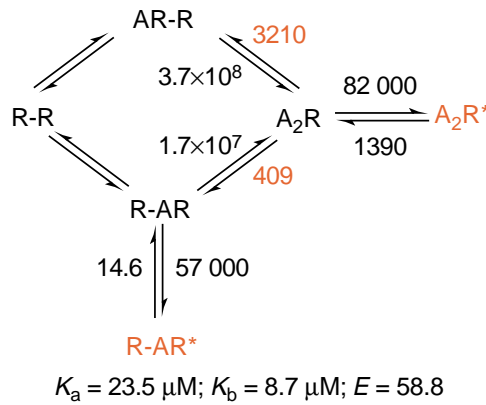

TRENDS in Neurosciences

Figure 3. Mechanisms for muscle nicotinic ACh receptors. There are two ligand-binding sites, and channels can open, albeit inefficiently, with only one agonist molecule (A) bound. Open states are marked ${ }^{*}$ and coloured red. (a) The two sites are identical in the unliganded state, although they can interact while still shut if the binding constant $K_{1}\left(=k_{-1} / k_{+1}\right)$ is not the same as $K_{2}\left(=k_{-2} / k_{+2}\right)$ (but the pre-opening conformation change that this implies is not included explicitly in the mechanism). (b-d) The two sites are different in the unliganded state. (b) A mechanism with two different sites: the two cylinders represent two $\alpha$ subunits $(\alpha \delta$ and $\alpha \varepsilon)$ and the red sphere represents an ACh molecule. There are two different monoliganded forms, both of which can open, although one produces only very short opening. Roughly representative channel opening events are shown. The opening at the top is $0.36 \mathrm{~ms}$, that at the bottom is $45 \mu \mathrm{s}$, and the centre insert shows a typical diliganded burst with duration of $\sim 4$ ms; it contains at least five openings separated by very brief shutting events. All openings seem to have the same amplitude, but the shortest ones are attenuated by the filter (c,d) The same mechanism, with values of the rate constants found using the HJCFIT program, for the wild-type nicotinic receptor (c) and for the slow channel myasthenic syndrome mutant $\varepsilon$ L221F (d) (values are in $\mathrm{s}^{-1}$, apart from association rate constants in $\mathrm{M}^{-1} \mathrm{~s}^{-1}$ ) [28]. These rate constants were found by simultaneous fit of several datasets, the fit being constrained to give a specified $\mathrm{EC}_{50}$ (measurement of agonist potency). Equilibrium constants for binding to the two sorts of site, and for diliganded gating, are shown below the mechanisms. The receptor is represented as $R-R$, but in full it should be shown as $R_{a}-R_{b}$ to indicate that the two sites are different (it is not known whether the $a$ site or the $b$ site corresponds with $\alpha \delta$ ). The two sites were assumed to be independent, so the binding to site $a$ is independent of whether site $b$ is occupied or not (rate constants on opposite sides of the cycle are therefore equal). The fitted mechanism included a rarely visited brief shut state, distal to the open state, which produces a slightly better fit for the wild type $[27,28]$. The biggest effect of the mutation is to decrease the total dissociation rate of ACh from diliganded receptors from $14300 \mathrm{~s}^{-1}$ for the wild type to $4600 \mathrm{~s}^{-1}$ for the mutants. The total dissociation rate (sum of the two rate constants for dissociation from diliganded receptors) can be found more accurately than either of the two separate dissociation rates [25]. The opening rate $\beta_{2}$ is also somewhat increased, and the shutting rate $\alpha_{2}$ is decreased. The effect of these changes is to predict a sixfold slowing of the synaptic current, much as is seen in patients with this mutation [28].

identical. The same possibility arises in Figure 3(b-d), although now there are separate values for the two different sites. If the second binding is tighter than the first $\left(K_{1}>K_{2}\right)$, this is usually described, somewhat confusingly, as 'cooperativity of binding'. Even when the affinity is the same for the first and second binding events $\left(K_{1}=K_{2}\right)$, the observed macroscopic response will have a Hill slope $>1$ ('cooperativity') as a consequence of the concerted conformation change to the open state. But the term 'cooperativity' is not very helpful when attempting to relate function and structure. Its definition, like that of 'allosteric', has become too vague for clarity [20]. The important point is that, if the binding affinity for a second molecule depends on whether or not one molecule is already bound, this implies that one binding site can sense whether or not the other site is occupied, while the channel is still shut.

In nicotinic receptors, the two binding sites probably do not interact while the channel is closed $[2,27,28]$. However, for homomeric glycine receptors, it is impossible to get a good fit to the data without postulating that at least the first binding event influences subsequent bindings [29,30]. Because the binding sites are far apart $(\sim 20 \AA$ in the snail ACh-binding protein), a direct electrostatic interaction is somewhat unlikely. The only obvious alternative way in which one site could influence another is because of a change in conformation that follows binding but occurs while the channel is still shut [29]. If such a conformation change occurs, it should be incorporated in the reaction mechanism. This has not usually been attempted, but one 


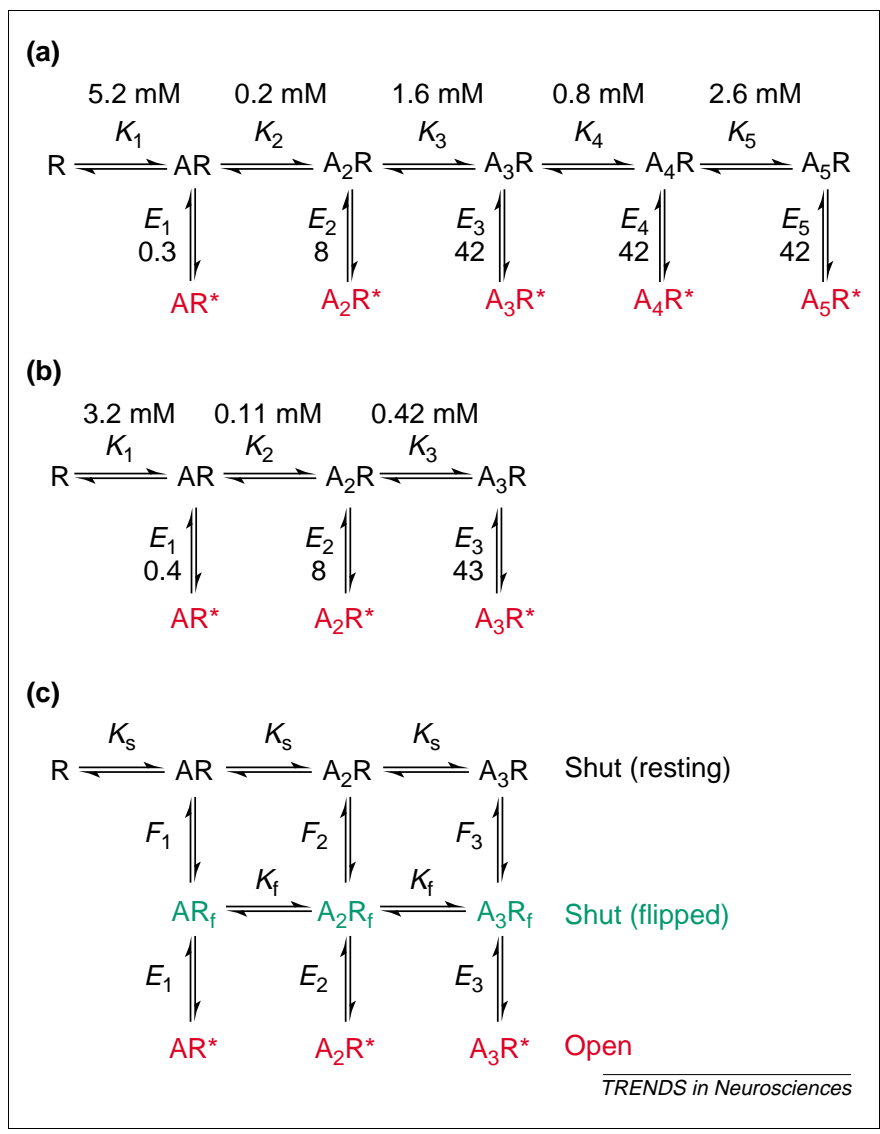

Figure 4. Mechanisms for glycine receptors. Panels (a) and (b) show two possible mechanisms for homomeric glycine receptors [29]; (c) shows a possible mechan ism for heteromeric glycine receptors that incorporates the possibility of a conformation change ('flip') occurring before the channel opens, as an explanation for the apparent interaction between binding sites while the channel is shut. The homomeric receptor is a pentamer, and the single-channel results can be fitted well if it is supposed there are five binding sites, as in (a), but only as long as the gating rate constants saturate after three molecules are bound, as indicated by the equal values of $E_{3}, E_{4}$ and $E_{5}$. In fact, an indistinguishable fit can be found even if only three binding sites are postulated, as in (b). Notice that the three binding constants $K_{1}, K_{2}$ and $K_{3}$ in (a) and (b) are not the same, which implies that the binding sites interact while the channel is shut. A pre-opening conformation change of the sort shown in (c) is a potential explanation for this observation. The channel changes conformation (equilibrium constant, $F$ ) to a 'flipped' form with higher agonist affinity before opening. Notice that the binding constants are now all the same for any specified conformation (denoted $K_{\mathrm{s}}$ for the resting shut state and $K_{\mathrm{f}}$ for the flipped shut state), independently of how many glycine molecules are already bound [39]. Here $E$ denotes the equilibrium constant for the open-shut conformation change $E=\beta / \alpha$, where $\beta$ is the rate constant for channel opening and $\alpha$ is the closing rate constant; the equilibrium binding constants are $K=k_{-} / k_{+}$. Rate constants were estimated by Beato et al. [29] but only the equilibrium constants are shown in (a) and (b), for clarity.

way of doing so for the heteromeric glycine receptor is illustrated in Figure 4(c), and single-channel results are consistent with something like this as an explanation for the observed subunit interaction [39].

It could be that the change in shape seen in structural studies of the binding site construct of AMPA glutamate receptors $[15,40]$ corresponds to such a pre-opening conformation change. This will be discussed here in the context of partial agonists.

There is little unanimity about reaction mechanisms for NMDA receptors. Simple schemes to account for single-channel results, at saturating concentrations only, have been proposed by Popescu and Auerbach [41]. There is no knowledge about whether subunit interactions occur while the channel is shut, but a reaction scheme that incorporates explicitly a pre-opening conformation change has been postulated by Banke et al. [42], and a more complete version of their mechanism that includes all binding steps is shown in Figure 5.

The number of shut states in this mechanism is, in principle, more than enough to account for the complex structure of the individual channel activations found in NMDA receptors $[43,44]$. However, uncertainties in how to incorporate the effects of $\mathrm{H}^{+}$and zinc, and various sorts of desensitization complicate the fitting of this sort of mechanism to single-channel data.

\section{Homomeric channels}

The only homomeric ion channel to have been analyzed in detail by single-channel methods is the glycine $\alpha 1$ receptor $[29,30]$. It seems likely that this homomeric pentamer would be symmetrical, and therefore that the five binding sites would be identical in the resting state, although crystallographic evidence is thin because of the paucity of protein structures with no ligand bound. Indeed, it was found that mechanisms (analogous with those in Figure $3 b-d$ ) with initially different, non-interacting sites could not provide a good fit to the observations.

Simple sequential binding mechanisms such as those in Figure 4 could provide a good fit to both single-channel data and the $P_{\text {open-concentration curve, but only if }}$ interaction between binding sites was allowed while the channel is still shut (e.g. $K_{1} \neq K_{2} \neq K_{2}$ ).

These results provide strong evidence for interactions between binding sites while the channel is still shut. Beato et al. [29] go further and suggest a slightly more complex model than that in Figure 4(a), based on the likely topology of binding, and with this it is possible to get a good fit by assuming that only the first binding influences other subunits. It remains to be seen whether the apparent interactions can be explained by a pre-opening conformation change of the sort postulated for the heteromeric receptor in Figure 4(c).

Perfectly good fits could be obtained by postulating either three (Figure 4b) or five (Figure 4a) binding sites, and these two cases could not be distinguished. What was clear was that, if there are indeed five binding sites, it must be supposed that the gating reaction 'saturates' after three agonist molecules are bound. If the 'efficacy' with $i$ ligand molecules bound is defined as $E_{\mathrm{i}}=\beta_{\mathrm{i}} / \alpha_{\mathrm{i}}$, then it is necessary to suppose that $E_{4}$ and $E_{5}$ are much the same as $E_{3}$ [29], as indicated by the numbers in Figure 4(a). Such saturation of gating might be rationalized in terms of structure by speculating that after three of the M2 domains of the $\alpha$ subunits have rotated, and broken out of the side-to-side bonds in the hydrophobic gate (Box 1), this ring is destabilized as much as possible. Thus, additional binding of a fourth and fifth agonist molecule, although opening the channel, does so no more effectively than binding of three. Surprisingly, at first sight, fast concentration jumps are not expected to discriminate between three and five binding events [29].

\section{The nature of partial agonists}

It was first suggested by del Castillo and Katz [45] that a partial agonist was one for which the gating equilibrium 


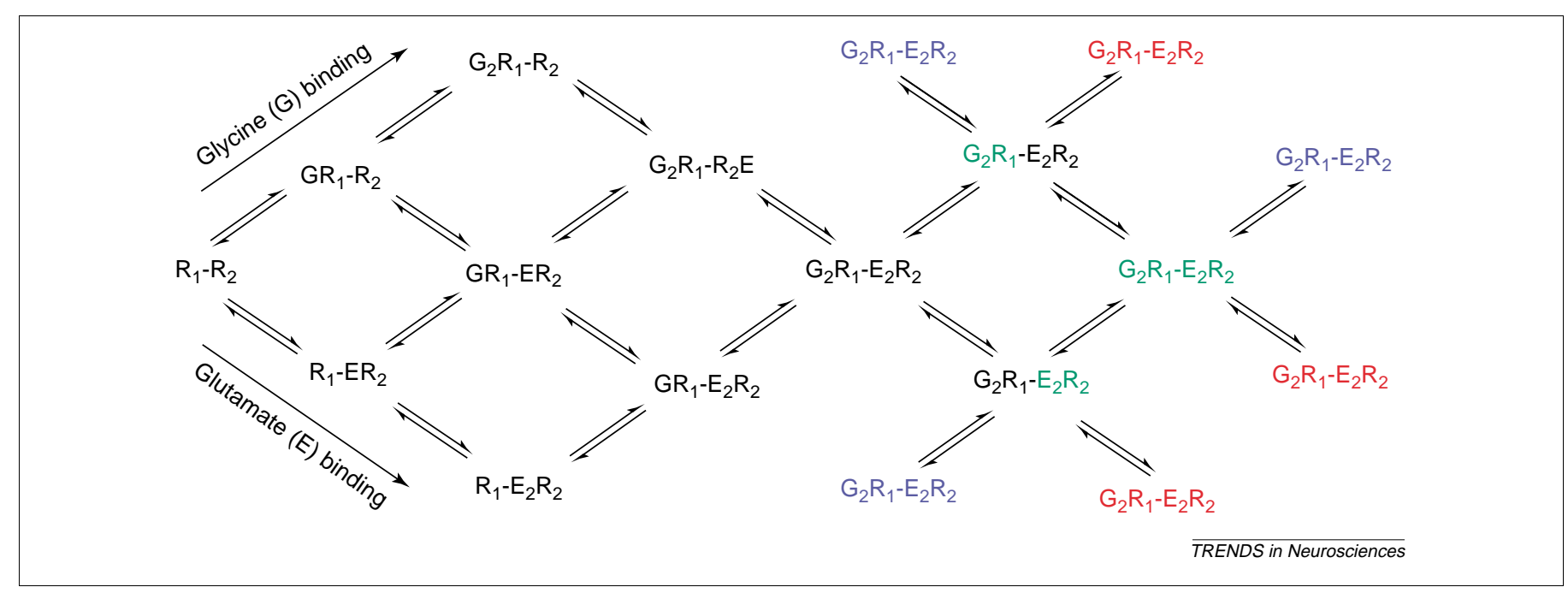

Figure 5. Postulated mechanisms for NMDA receptors. The NMDA receptor is supposed to consist of an NR1 subunit dimer ( $\left.R_{1}\right)$ that can bind two glycine molecules (G), and an NR2 subunit dimer $\left(R_{2}\right)$ that can bind two glutamate molecules (E). No opening occurs until all four sites are occupied, at which point either the NR1 or the NR2 dimer can undergo a conformation change while still shut, to a pre-open conformation (green). From this state, the channel can either open (red) or desensitize (blue). It has not yet proved possible to estimate rate constants for a mechanism of this complexity.

constant $E=\beta / \alpha$ is small, so the maximum possible response $E /(1+E)$ (i.e. the maximum fraction of open channels) is well short of 1 . This of course begs the question of what structural features determine the value of $E$, but we are a long way from being able to predict that from first principles. For most receptors, Katz's explanation is likely to be essentially right. For example, on the muscle nicotinic receptor, $\mathrm{ACh}$ is normally very efficacious $(E \approx 30$, maximum response $\sim 97 \%$ for diliganded channels). But at positive membrane potentials, $E$ is much reduced (from $\sim 30$ to $\sim 0.7$, largely as a result of increased shutting rate), so ACh becomes a frank partial agonist with a maximum response of $\sim 41 \%$ [46]. A good example of a partial agonist is choline, which has a gating equilibrium constant of $E_{2} \approx 0.05$, largely because of a much ( 200-fold) slower opening rate constant than ACh [47]. The investigation of efficacy at normal membrane potentials is hindered by the fact that all nicotinic agonists block the channel as well as opening it.

In Katz's framework, a partial agonist for an ion channel would be one that produced an open probability of substantially less than 1 when it occupies all receptors. This would result in a smaller maximum macroscopic response, despite the single-channel conductance being the same for all agonists. A possible alternative mechanism is that a partial agonist might selectively open channels of low conductance, thus producing a small response even if the agonist achieved an open probability near 1 on these receptors. The evidence so far favours Katz's interpretation of partial agonism for all ion channels with the single exception of the AMPA-type glutamate receptor.

In the case of the nicotinic receptor, the conductance of the open channel is independent of the nature of the agonist [48], and independent of the number of agonist molecules that are bound. This is consistent with the idea that the structure of the open channel is much the same regardless of how it is caused to open, as although there was a concerted transition to a single open conformation.
The same seems to be true for glycine and $\mathrm{GABA}_{\mathrm{A}}$ receptors $[49,50]$.

NMDA receptors present much bigger problems of interpretation. The NMDA receptor (NR1-NR2a) is open for only approximately one third of the time when glutamate is bound (in the absence of magnesium) [44,51] which, on the face of it, means that glutamate itself is a partial agonist. However, it is likely that the relatively low maximum $P_{\text {open }}$ could result from block by $\mathrm{H}^{+}$, and perhaps by contaminant $\mathrm{Zn}^{2+}[52,53]$. It seems that the NMDA receptor resembles others in that the singlechannel conductance is independent of the nature of the agonist that acts at the glutamate sites [54,55]. The general structure of the binding site is similar to that of AMPA receptors (Figure 2). Crystallographic studies have been done on a glycine-binding-site construct made from the NR1 subunit [16]. In contrast to the case of AMPA receptors, a partial agonist at this site caused as much domain closure as a full agonist, but so far it has not proved possible to explain partial agonism in terms of gating constant(s).

There is more information about AMPA receptors, for which partial agonists have been well characterized at least at the level of macroscopic concentration-response curves. It seems that, in contrast to any of the other receptors mentioned here, the number of agonist molecules that are bound $[56,57]$ and the chemical nature of the agonist [40] do influence (average) channel conductance, by altering the relative numbers of openings that occur at each of several subconductance levels. Structures of the ligand-binding construct from GluR2 show that the extent of closure of the 'clamshell' is smaller for partial agonists than for full agonists [40], although the extent to which this represents an opening, or a pre-opening, conformation change is unknown, because the structures are determined under conditions in which an intact receptor would be desensitized. Neither is the maximum open probability for each conductance level known. This phenomenon, whatever the details, appears to be different 
from that observed in nicotinic or even NMDA receptors, none of which shows subconductance levels that depend on the number or the nature of bound ligands.

Of course, it is possible that the pentameric receptors could show a pre-opening conformation that depended on the nature of the agonist (there is no information about this), and that this was followed by a concerted transition to an open conformation that is similar for all agonists. A variable pre-opening conformation change could destabilize the shut conformation to various extents, leading to values for the opening rate constant $\beta$ that depend on the nature of the agonist but that lead to an open conformation that is similar for all agonists.

\section{Conclusions and future work}

Progress is being made rapidly but there is a long way to go. There are still no high-resolution crystal structures of entire receptors in the shut and open conformations, so the field is still well behind the position that haemoglobin reached in 1960s. Large numbers of mutations have been made, but even those that have been analyzed in detail (some are reviewed in Ref. [2]) often do not make much sense in our present state of knowledge. We are a long way from being able to explain (much less predict) the effects of mutations such as nicotinic $\alpha \mathrm{N} 217 \mathrm{~K}$ [36] and $\varepsilon \mathrm{L} 221 \mathrm{~F}$ [28], which affect mainly agonist-binding despite being in or close to M1, a long distance from the binding region. At present, making sense of structure-function in proteins is a bit like structure-activity in pharmacology: we do too much viewing of observations through rose-tinted retrospectacles, and we have too little predictive power. No doubt that will improve in the fullness of time.

\section{References}

1 Fersht, A.R. (1995) Characterizing transition states in protein folding: an essential step in the puzzle. Curr. Opin. Struct. Biol. 5, 79-84

2 Colquhoun, D. et al. (2003) Nicotinic acetylcholine receptors. In Burger's Medicinal Chemistry Drug Discovery and Drug Development, 6th edn, (Abraham, D., ed.), pp. 357-405, John Wiley

3 Auerbach, A. (2003) Life at the top: the transition state of AChR gating. Sci. STKE 2003, re11

4 Engel, A.G. et al. (2003) Congenital myasthenic syndromes: progress over the past decade. Muscle Nerve 27, 4-25

5 Unwin, N. (2003) Structure and action of the nicotinic acetylcholine receptor explored by electron microscopy. FEBS Lett. 555, 91-95

6 Miyazawa, A. et al. (2003) Structure and gating mechanism of the acetylcholine receptor pore. Nature 424, 949-955

7 Brejc, K. et al. (2001) Crystal structure of an ACh-binding protein reveals the ligand-binding domain of nicotinic receptors. Nature 411 , 269-276

8 Celie, P.H. et al. (2004) Nicotine and carbamylcholine binding to nicotinic acetylcholine receptors as studied in AChBP crystal structures. Neuron 41, 907-914

9 Langosch, D. et al. (1988) Conserved quaternary structure of ligandgated ion channels: The postsynaptic glycine receptor is a pentamer. Proc. Natl. Acad. Sci. U. S. A. 85, 7394-7398

10 Kuhse, J. et al. (1993) Assembly of the inhibitory glycine receptor: identification of amino acid sequence motifs governing subunit stoichiometry. Neuron 11, 1049-1056

11 Burzomato, V. et al. (2003) Stoichiometry of recombinant heteromeric glycine receptors revealed by a pore-lining region point mutation. Receptors Channels 9, 353-361

12 Laube, B. et al. (1997) Molecular determinants of agonist discrimination by NMDA receptor subunits: Analysis of the glutamate binding site on the NR2B subunit. Neuron 18, 493-503

13 Anson, L.C. et al. (1998) Identification of amino acid residues of the
NR2A subunit which control glutamate potency in recombinant NR1/ NR2A NMDA receptors. J. Neurosci. 18, 581-589

14 Schorge, S. and Colquhoun, D. (2003) Studies of NMDA receptor function and stoichiometry with truncated and tandem subunits. J. Neurosci. 23, 1151-1158

15 Armstrong, N. and Gouaux, E. (2000) Mechanisms for activation and antagonism of an AMPA-sensitive glutamate receptor: crystal structures of the GluR2 ligand binding core. Neuron 28, 165-181

16 Furukawa, H. and Gouaux, E. (2003) Mechanisms of activation, inhibition and specificity: crystal structures of the NMDA receptor NR1 ligand-binding core. EMBO J.22, 2873-2885

17 Grosman, C. et al. (2000) Mapping the conformational wave of acetylcholine receptor channel gating. Nature 403, 773-776

18 Cymes, G.D. et al. (2002) Structure of the transition state of gating in the acetylcholine receptor channel pore: a $\phi$-value analysis. Biochemistry $41,5548-5555$

19 Chakrapani, S. et al. (2003) The role of loop 5 in acetylcholine receptor channel gating. J. Gen. Physiol. 122, 521-539

20 Colquhoun, D. (1998) Binding, gating, affinity and efficacy. The interpretation of structure-activity relationships for agonists and of the effects of mutating receptors. Br. J. Pharmacol. 125, 924-947

21 Colquhoun, D. and Sakmann, B. (1981) Fluctuations in the microsecond time range of the current through single acetylcholine receptor ion channels. Nature 294, 464-466

22 Colquhoun, D. and Sakmann, B. (1985) Fast events in single-channel currents activated by acetylcholine and its analogues at the frog muscle end-plate. J. Physiol. 369, 501-557

23 Qin, F. et al. (1996) Estimating single-channel kinetic parameters from idealized patch-clamp data containing missed events. Biophys. J. 70, $264-280$

24 Colquhoun, D. et al. (1996) Joint distributions of apparent open times and shut times of single ion channels and the maximum likelihood fitting of mechanisms. Philos. Trans. R. Soc. Lond. B Biol. Sci. 354, $2555-2590$

25 Colquhoun, D. et al. (2003) The quality of maximum likelihood estimates of ion channel rate constants. J. Physiol. 547, 699-728

26 Magleby, K.L. and Weiss, D.S. (1990) Identifying kinetic gating mechanisms for ion channels by using two- dimensional distributions of simulated dwell times. Proc. R. Soc. Lond. B. Biol. Sci. 241, 220-228

27 Salamone, F.N. et al. (1999) A re-examination of adult mouse nicotinic acetylcholine receptor channel activation kinetics. J. Physiol. 516, 315-330

28 Hatton, C.J. et al. (2003) Properties of the human muscle nicotinic receptor, and of the slow-channel myasthenic syndrome mutant عL221F, inferred from maximum likelihood fits. J. Physiol. 547, $729-760$

29 Beato, M. et al. (2004) The activation mechanism of $\alpha 1$ homomeric glycine receptors. J. Neurosci. 24, 895-906

30 Beato, M. et al. (2002) Openings of the rat recombinant $\alpha 1$ homomeric glycine receptor as a function of the number of agonist molecules bound. J. Gen. Physiol. 119, 443-466

31 Grosman, C. and Auerbach, A. (2001) The dissociation of acetylcholine from open nicotinic receptor channels. Proc. Natl. Acad. Sci. U. S. A. 98, 14102-14107

32 Sine, S.M. et al. (1990) Activation of Torpedo acetylcholine receptors expressed in mouse fibroblasts: single channel current kinetics reveal distinct agonist binding affinities. J. Gen. Physiol. 96, 395-437

33 Jackson, M.B. (1988) Dependence of acetylcholine receptor channel kinetics on agonist concentration in cultured mouse muscle fibres. J. Physiol. 397, 555-583

34 Zhang, Y. et al. (1995) Activation of recombinant mouse acetylcholine receptors by acetylcholine, carbamylcholine and tetramethylammonium. J. Physiol. 486, 189-206

35 Auerbach, A. et al. (1996) Voltage dependence of mouse acetylcholine receptor gating: different charge movements in di-, mono- and unliganded receptors. J. Physiol. 494, 155-170

36 Wang, H.L. et al. (1997) Mutation in the M1 domain of the acetylcholine receptor $\alpha$ subunit decreases the rate of agonist dissociation. J. Gen. Physiol. 109, 757-766

37 Milone, M. et al. (1997) Slow-channel myasthenic syndrome caused by enhanced activation, desensitization, and agonist binding affinity attributable to mutation in the M2 domain of the acetylcholine receptor $\alpha$ subunit. J. Neurosci. 17, 5651-5665 
38 Monod, J. et al. (1965) On the nature of allosteric transitions: a plausible model. J. Mol. Biol. 12, 88-118

39 Burzomato, V. et al. (2004) Activation of heteromeric $\alpha 1 / \beta$ rat glycine receptors. Biophysical Society Abstracts 1497 (http://www.biophysics. org/abstracts/)

40 Jin, R. et al. (2003) Structural basis for partial agonist action at ionotropic glutamate receptors. Nat. Neurosci. 6, 803-810

41 Popescu, G. and Auerbach, A. (2003) Modal gating of NMDA receptors and the shape of their synaptic response. Nat. Neurosci. 6, 476-483

42 Banke, T.G. and Traynelis, S.F. (2003) Activation of NR1/NR2B NMDA receptors. Nat. Neurosci. 6, 144-152

43 Gibb, A.J. and Colquhoun, D. (1991) Glutamate activation of a single NMDA receptor-channel produces a cluster of channel openings. Proc. R. Soc. Lond. B. Biol. Sci. 243, 39-45

44 Wyllie, D.J.A. et al. (1998) Single-channel activations and concentration jumps: comparison of recombinant NR1/NR2A and NR1/NR2D NMDA receptors. J. Physiol. 510, 1-18

45 del Castillo, J. and Katz, B. (1957) Interaction at end-plate receptors between different choline derivatives. Proc. R. Soc. Lond. B. Biol. Sci. $146,369-381$

46 Colquhoun, D. and Ogden, D.C. (1988) Activation of ion channels in the frog end-plate by high concentrations of acetylcholine. J. Physiol. 395, 131-159

47 Grosman, C. and Auerbach, A. (2000) Asymmetric and independent contribution of the second transmembrane segment $12^{\prime}$ residues to diliganded gating of acetylcholine receptor channels. A single-channel study with choline as the agonist. J. Gen. Physiol. 115, 637-651

48 Gardner, P. et al. (1984) Conductances of single ion channels opened by nicotinic agonists are indistinguishable. Nature 309, 160-162

49 Lewis, T.M. et al. (2003) Kinetic determinants of agonist action at the recombinant human glycine receptor. J. Physiol. 549, 361-374

50 Mortensen, M. et al. (2004) Activation of single heteromeric GABAA receptor ion channels by full and partial agonists. J.Physiol. [DOI: 10.1113/jphysiol.2003.054734]

51 Chen, N. et al. (1999) Subtype-dependence of NMDA receptor channel open probability. J. Neurosci. 19, 6844-6854

52 Low, C.M. et al. (2003) Molecular determinants of proton-sensitive $\mathrm{N}$-methyl-D-aspartate receptor gating. Mol. Pharmacol. 63, $1212-1222$

53 Traynelis, S.F. and Cull-Candy, S.G. (1990) Proton inhibition of $\mathrm{N}$-methyl-D-aspartate receptors in cerebellar neurons. Nature 345 , $347-350$

54 Howe, J.R. et al. (1991) Currents through single glutamate-receptor channels in outside-out patches from rat cerebellar granule cells. J. Physiol. 432, 143-202

55 McLarnon, J.G. and Sawyer, D. (1993) Dependence of single channel properties of the N-methyl-D-aspartate ion channel on stereoisomer agonists. Exp. Brain Res. 95, 8-14

56 Rosenmund, C. et al. (1998) The tetrameric structure of a glutamate receptor channel. Science 280, 1596-1599

57 Smith, T.C. and Howe, J.R. (2000) Concentration-dependent substate behavior of native AMPA receptors. Nat. Neurosci. 3, 992-997

58 Unwin, N. et al. (2002) Activation of the nicotinic acetylcholine receptor involves a switch in conformation of the $\alpha$ subunits. J. Mol. Biol. 319, 1165-1176

59 Gouaux, E. (2004) Structure and function of AMPA receptors J. Physiol. 554, 249-253

60 Shen, X.M. et al. (2003) Mutation causing severe myasthenia reveals functional asymmetry of AChR signature cystine loops in agonist binding and gating. J. Clin. Invest. 111, 497-505

61 Grosman, C. et al. (2000) The extracellular linker of muscle acetylcholine receptor channels is a gating control element. J. Gen. Physiol. 116, 327-339

62 Rajendra, S. et al. (1995) Mutation of an arginine residue in the human glycine receptor transforms $\beta$-alanine and taurine from agonists into competitive antagonists. Neuron 14, 169-175

63 Lynch, J.W. et al. (1997) Identification of intracellular and extracellular domains mediating signal transduction in the inhibitory glycine receptor chloride channel. EMBO J. 16, 110-120

64 Lewis, T.M. et al. (1998) Properties of human glycine receptors containing the hyperekplexia mutation $\alpha 1(\mathrm{~K} 276 \mathrm{E})$, expressed in Xenopus oocytes. J. Physiol. 507, 25-40

65 Shan, Q. et al. (2003) Asymmetric contribution of $\alpha$ and $\beta$ subunits to the activation of $\alpha \beta$ heteromeric glycine receptors. J. Neurochem. 86, 498-507

66 Revah, F. et al. (1991) Mutations in the channel domain alter desensitization of a neuronal nicotinic receptor. Nature 353, 846-849

67 Labarca, C. et al. (1995) Channel gating governed symmetrically by conserved leucine residues in the M2 domain of nicotinic receptors. Nature 376, 514-516

68 Chang, Y. et al. (1996) Stoichiometry of a recombinant GABAA receptor. J. Neurosci. 16, 5415-5424

69 Boorman, J.P. et al. (2000) Stoichiometry of human recombinant neuronal nicotinic receptors containing the $\beta 3$ subunit expressed in Xenopus oocytes. J. Physiol. 529, 565-577

70 Wilson, G.G. and Karlin, A. (1998) The location of the gate in the acetylcholine receptor channel. Neuron 20, 1269-1281

\title{
Articles of interest in other Trends journals
}

\author{
Motor neurons rely on motor proteins \\ Erika L. F. Holzbaur, Trends in Cell Biology 10.1016/j.tcb.2004.03.009 \\ The brain circuitry of attention \\ Stewart Shipp, Trends in Cognitive Sciences 10.1016/j.tics.2004.03.004
}

Mining event-related brain dynamics

Scott Makeig, Stefan Debener, Julie Onton and Arnaud Delorme, Trends in Cognitive Sciences 10.1016/j.tics.2004.03.008

Disfluencies and human language comprehension

Fernanda Ferreira and Karl G. D. Bailey, Trends in Cognitive Sciences 10.1016/j.tics.2004.03.011

The ups and downs of addiction: role of metabotropic glutamate receptors

Paul J. Kenny and Athina Markou, Trends in Pharmacological Sciences 10.1016/j.tips.2004.03.009

Neuroprotective strategies for Parkinson's disease: conceptual limits of animal models and clinical trials

Wassilios Meissner, Michael P. Hill, François Tison, Christian E. Gross and Erwan Bezard, Trends in Pharmacological Sciences 10.1016/j.tips.2004.03.003 